\title{
A Phylogenomic View of Ecological Specialization in the Lachnospiraceae, a Family of Digestive Tract-Associated Bacteria
}

\author{
Conor J. Meehan ${ }^{1,2}$ and Robert G. Beiko ${ }^{2, *}$ \\ ${ }^{1}$ Department of Biochemistry and Molecular Biology, Dalhousie University, Halifax, Nova Scotia, Canada \\ ${ }^{2}$ Faculty of Computer Science, Dalhousie University, Halifax, Nova Scotia, Canada \\ *Corresponding author: E-mail: beiko@cs.dal.ca.
}

Accepted: March 4, 2014

\begin{abstract}
Several bacterial families are known to be highly abundant within the human microbiome, but their ecological roles and evolutionary histories have yet to be investigated in depth. One such family, Lachnospiraceae (phylum Firmicutes, class Clostridia) is abundant in the digestive tracts of many mammals and relatively rare elsewhere. Members of this family have been linked to obesity and protection from colon cancer in humans, mainly due to the association of many species within the group with the production of butyric acid, a substance that is important for both microbial and host epithelial cell growth. We examined the genomes of 30 Lachnospiraceae isolates to better understand the origin of butyric acid capabilities and other ecological adaptations within this group. Butyric acid production-related genes were detected in fewer than half of the examined genomes with the distribution of this function likely arising in part from lateral gene transfer (LGT). An investigation of environment-specific functional signatures indicated that human gut-associated Lachnospiraceae possess genes for endospore formation, whereas other members of this family lack key sporulationassociated genes, an observation supported by analysis of metagenomes from the human gut, oral cavity, and bovine rumen. Our analysis demonstrates that adaptation to an ecological niche and acquisition of defining functional roles within a microbiome can arise through a combination of both habitat-specific gene loss and LGT.
\end{abstract}

Key words: lateral gene transfer, microbial genomes, metagenomics, phylogenomics, butyric acid, sporulation.

\section{Introduction}

Mammal-associated microbiomes have been shown to influence host health and behavior (Cryan and O'Mahony 2011; Kinross et al. 2011; Muegge et al. 2011) and appear to be hotbeds for lateral gene transfer (LGT) (Smillie et al. 2011; Meehan and Beiko 2012). Lachnospiraceae is a family of clostridia that includes major constituents of mammalian gastrointestinal (Gl) tract microbiomes, especially in ruminants (Kittelmann et al. 2013) and humans (Gosalbes et al. 2011). The family is currently described in the National Center for Biotechnology Information (NCBI) taxonomy as comprising 24 named genera and several unclassified strains (Sayers et al. 2010) that share 165 ribosomal RNA gene (henceforth referred to as 16S) similarity (Bryant 1986; Dworkin and Falkow 2006). All known family members are strictly anaerobic (Dworkin and Falkow 2006), reside mainly within the digestive tracts of mammals (Bryant 1986; Downes et al. 2002; Carlier et al. 2004; Moon et al. 2008), and are thought to be primarily nonspore forming (Dworkin and Falkow 2006). Several members play key roles within the human Gl microbiome, demonstrated by their inclusion in an artificial bacterial community that has been used to repopulate a gut microbiome and remedy Clostridium difficile infections (Petrof et al. 2013). Early blooms of Lachnospiraceae may be linked with obesity (Cho et al. 2012), most likely due to their shortchain fatty acid (SCFA) production (Duncan et al. 2002). However, despite their apparent importance, little is known about the group as a whole outside of its use as an indicator of fecal contamination in water and sewage (Newton et al. 2011; McLellan et al. 2013) and the abundance of butyric acid-producing species within the group (Bryant 1986; Duncan et al. 2002; Louis et al. 2004, 2010; Charrier et al. 2006).

Butyric acid (also known as butanoic acid, butanoate, and butyrate) is an SCFA whose production prevents the growth of some microbes within the digestive tract (Zeng et al. 1994; 
Sun et al. 1998) and provides a source of energy for other microbes (Liu et al. 1999) and host epithelial cells (Roediger 1980; Mclntyre et al. 1993; Hague et al. 1996; Pryde et al. 2002). Butyrate also regulates expression of the AP-1 signaling pathway in key components of human physiology (Nepelska et al. 2012). These functions link butyric acid to protection against colon cancer (Hague et al. 1996; Mandal et al. 2001) and a potential influence on obesity levels (Duncan et al. 2008; Turnbaugh et al. 2008). Two pathways are responsible for fermentation of this SCFA: through butyrate kinase or through butyryl-CoA:acetate CoA-transferase (BCoAT) (Walter et al. 1993; Duncan et al. 2002). This production appears to be restricted mainly to organisms within the class Clostridia (Louis et al. 2010) and has been demonstrated in many strains of Lachnospiraceae (Attwood et al. 1996; Duncan et al. 2002; Charrier et al. 2006; Kelly et al. 2010; Louis et al. 2010).

Although the production of butyrate links many Lachnospiraceae to key roles within digestive tract microbiomes, it is not known why only some members produce this SCFA and what the ecological role of the remaining family members might be. Here, we investigate the relationship between phylogeny, ecology, and biochemistry in this group by examining a set of 30 sequenced genomes, combined with marker gene surveys from a wide range of habitats and metagenomic samples collected from the habitats with high numbers of Lachnospiraceae. Endospore formation distinguished Lachnospiraceae from different habitats, with complete or near-complete sporulation pathways in human gutassociated microorganisms, and many key pathways absent from other members of the group. Although endospore formation capability appeared to be a result of habitat-specific loss, the distribution of butyrate production capabilities showed strong evidence of LGT. The fluidity of butyrate production and other properties highlights a range of evolutionary processes that impact on adaptation and host interactions.

\section{Materials and Methods}

\section{Assessing the Habitat of Lachnospiraceae Members}

A determination of the environmental range of members of the Lachnospiraceae was undertaken using a phylogenetic assignment method. All 165 sequences from completed genomes and all Clostridiales-type strains in the Ribosomal Database Project (Cole et al. 2009) were aligned to the Greengenes reference alignment template using PyNAST (Caporaso et al. 2010) and masked to include only the phylogenetically informative sites, resulting in an alignment of 2,217 sequences and 1,287 sites. A reference tree was then created from these sequences using RaxML version 7.2.5 (Stamatakis 2006) with a GTR $+\Gamma$ model. Presence within a habitat was assessed by aligning reads from 1,697 environmental samples of $16 \mathrm{~S}$ sequences from MG-RAST
(Meyer et al. 2008), sorted into 17 habitat types (supplementary table S1, Supplementary Material online), added to the reference alignment using PyNAST, and placed on the reference tree using pplacer version 1.1.alpha13 (Matsen et al, 2010). Taxonomic classification of reads was then undertaken using the classify function of guppy, a part of the pplacer package. Reads were classified as a given taxonomic rank if the posterior probability of that assignment was above 0.7. The percentage of classified reads assigned to Lachnospiraceae was calculated per sample and then aggregated between samples into broad habitat definitions.

\section{Butyric Acid Production}

Sequenced genomes identified as Lachnospiraceae were retrieved from NCBI on April 18, 2012 (supplementary table S2, Supplementary Material online). This resulted in 30 genomes ( 2 completed and 28 permanent draft) from four primary habitats: the human digestive tract, cow rumen, human oral cavity, and sediment containing paper-mill and domestic waste. The potential for butyric acid production was then assessed within each Lachnospiraceae sequenced genome. Sequences annotated as butyrate kinase were retrieved from the KEGG database, version 58.1 (Kanehisa et al. 2004), as this encodes one of the final steps of the two butyric acid pathways. The other path to butyric acid production is through utilization of BCoAT (Louis et al. 2010). The sequences derived from Louis et al. (2010) constituted the reference database for our search. These two data sets were used to mine the protein sets of each sequenced Lachnospiraceae genome using USEARCH 4.0.38 (Edgar 2010) with an e-value cut off of $10^{-30}$ and a minimum identity cut off of $70 \%$. The origin of the butyrate-related genes was assessed using a phylogenetic approach. Protein sequences encoded by 3,500 bacterial and archaeal genomes were retrieved from $\mathrm{NCBI}$, and USEARCH was used in same manner as above to search for the two butyric acid-related genes, with the Lachnospiraceae sequences identified above as queries. Sequences were aligned using MUSCLE version 3.8.31 (Edgar 2004a, 2004b) and trimmed using BMGE version 1.1 (Criscuolo and Gribaldo 2010) with a BLOSUM30 matrix and a 0.7 entropy cut off. A phylogenetic tree was created using FastTree version 2.1.4 (Price et al. 2010) with a GTR model and a gamma parameter to model rate variation across sites.

To test whether LGT occurred within the history of these genes, a comparison of the resulting topologies to the $16 \mathrm{~S}$ tree (as a proxy for implied vertical inheritance) was undertaken. The longest $16 \mathrm{~S}$ sequence from each genome found to have a predicted butyrate kinase was extracted, and an alignment and tree were built as above. The per-site likelihoods of the $16 \mathrm{~S}$ topology and the topology based on the butyrate kinase alignment were calculated using FastTree with the butyrate kinase alignment as the data set, and an approximately 
unbiased (AU) test was performed using CONSEL (Shimodaira and Hasegawa 2001). This procedure was repeated using the BCOAT-containing species.

\section{Clustering of Genomes Based on Homologous Gene Groups}

A comparative genomics approach was undertaken to understand the shared functional repertoires of members of the Lachnospiraceae. To construct a set of shared homologous protein-coding genes, BLASTClust (Altschul et al. 1990) was employed with a minimum match criterion of $40 \%$ identity and $70 \%$ length on all genes. Functional assignment to each cluster was performed using the Clusters of Orthologous Groups (COG) database (Tatusov et al. 2000). BlastP (Altschul et al. 1990) with a $10^{-3}$ e-value cut off was employed for each gene cluster using representative protein sequences for each of the 18 COG functional categories as a database. Lachnospiraceae genomes were then clustered based on pair-wise counts of shared homologous gene clusters to look for associations between shared genome content and habitat. These pair-wise counts were calculated using a normalized Hamming distance, such that the distance between genomes $x$ and $y$ is $(A+B-2 S) /(A+B)$ where $A$ and $B$ are the total gene counts of $x$ and $y$, respectively, and $S$ is the number of shared genes between $x$ and $y$ (Lin and Gerstein 2000). If a cluster contained more than one gene in a given genome (e.g., in-paralogs), $S$ equals the smaller gene count per genome. Counts were then clustered and displayed using the R package gplots (Warnes et al. 2012). Groups of interest were further analyzed using Interproscan version 4.8 (Zdobnov and Apweiler 2001) to determine what functions may define such groups.

\section{Distribution of Sporulation Capabilities in Sequenced Genomes and Metagenomes}

Each Lachnospiraceae genome was compared with the sporulation-associated proteins as found within Bacillus subtilis strain 168 (Kunst et al. 1997). The B. subtilis proteins labeled as within the main sporulation-associated families cot, spol, sps, and ssp were used as a database for a BlastP search with a $10^{-30}$ e-value cut off and all Lachnospiraceae proteins as queries. The putative history of each sporulation protein was assessed with the same phylogenetic method as was used for the butyric acid-related proteins.

Metagenomes for the human digestive tract (Yatsunenko et al. 2012; MG-RAST project 401; 107 samples), human oral cavity (Human Microbiome Project; MG-RAST project 385; 12 samples), and cow rumen (Brulc et al. 2009; MG-RAST project 24; four samples; Hess et al. 2011; SRA023560; one sample) were used to assess the distribution of Lachnospiraceaederived sporulation proteins in culture-independent data sets. The Lachnospiraceae-associated sporulation genes were used as a database with a metagenome sample as a query input to USEARCH with a $10^{-10}$ e-value cut off. From this set of results, we removed all reads whose best match was to a non-Lachnospiraceae genome in the set of 3,500 NCBI genomes. Final counts of reads designated as sporulationassociated were compared between habitats using STAMP version 2 (Parks and Beiko 2010) with a two-sided Welch's t-test and Bonferroni multiple test correction.

\section{Phylogenomic Analysis of the Lachnospiraceae}

Assessment of intra-family relationships was undertaken using three different methods: phylogenetic tree inference using 16S, tree inference using a concatenated alignment of 91 shared protein-coding genes, and a consensus network of relationships (Holland et al. 2004) based on the same set of shared genes.

All 16S rRNA gene sequences over 1,000 nucleotides long from each Lachnospiraceae genome along with those of two species from the family Ruminococcaceae as an outgroup (Ruminococcus albus 7 and Ethanoligenens harbinense YUAN-3) were aligned using PyNAST and trimmed to include only the phylogenetically informative sites used by Greengenes (DeSantis et al. 2006). A reference tree was created using RaxML version 7.2.5 with the evolutionary model $\mathrm{GTR}+\Gamma+\mathrm{I}$ as selected using the Bayesian information criterion in PartitionFinder (Lanfear et al. 2012). A set of familywide shared genes was created from the homologous gene clusters output from BlastClust. Those gene sets that were present as a single copy in each genome were selected, and orthology was confirmed using an all versus all BlastP between Lachnospiraceae proteomes with an e value of $10^{-10}$. USEARCH was used with an e-value cut off of $10^{-30}$ to find genes in the completed genomes of members of the Ruminococcaceae that would serve as an outgroup to these family-wide genes. Alignments were constructed using MUSCLE and trimmed using BMGE as above. Resulting alignments were then concatenated and a tree inferred using FastTree with a gamma parameter.

SEQBOOT (Felsenstein 1989) was used to generate 100 randomizations each of the 165 and concatenated alignments, which were then subjected to phylogenetic analysis as above to establish bootstrap support. Concordance between the $16 \mathrm{~S}$ tree and concatenated alignment tree was tested using the subtree prune-and-regraft (SPR) distance with rSPR version 1.2.0 (Whidden et al. 2010) and the AU test in CONSEL version 0.20 (Shimodaira and Hasegawa 2001). Individual gene alignments were also tested for concordance with the concatenated sequence tree using the $\mathrm{AU}$ test in CONSEL and with each other using rSPR. The set of shared Lachnospiraceae protein-coding genes was used to create a consensus network using SplitsTree4 (Huson and Bryant 2006) with a 0.7 similarity cut off and edges weighted by counts. 


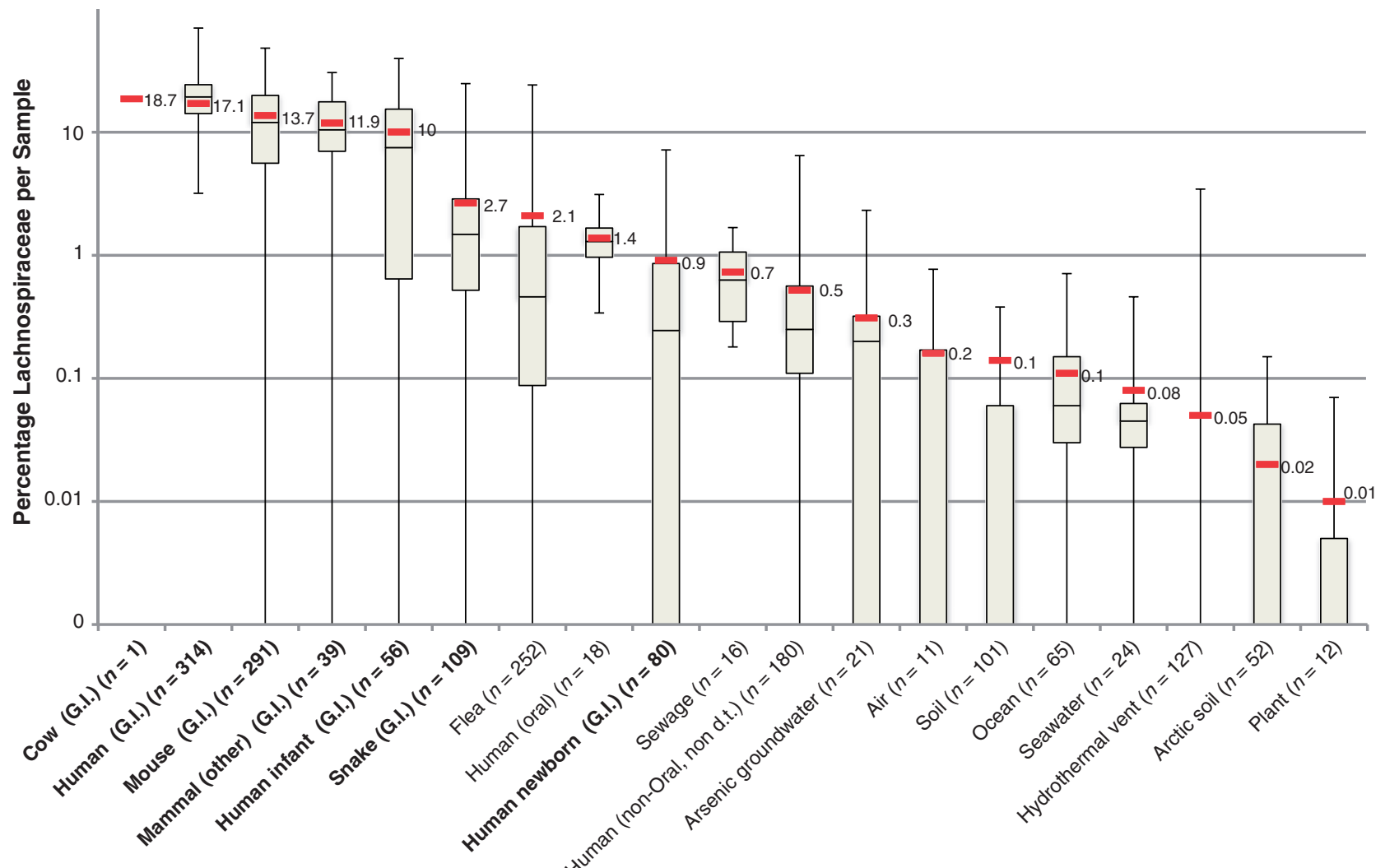

Habitat class

FIG. 1.-Environmental distribution of the Lachnospiraceae. A total of $2516 \mathrm{~S}$ rRNA gene surveys containing a total of 1,697 samples covering 17 different habitat classes were taxonomically profiled to identify the overall percentage of Lachnospiraceae. Boxplots outline the 25th, 50th, and 75th percentiles of the data. The minimum, maximum, and average (red box) percent abundance per sample of this family are also indicated. The number of samples per environment is listed beside habitat type and in supplementary table S1, Supplementary Material online. Each GI tract-associated habitat is highlighted in bold.

\section{Results}

Lachnospiraceae Are Common Only in Host-Associated and Sewage Effluent Samples

We examined a total of 1,697 published marker gene surveys from different environments to determine the primary habitats of the Lachnospiraceae. Sequences associated with the group were more abundant in the Gl tracts of mammals compared with other environments, including other mammal-associated body sites (fig. 1). Although mammalian Gl samples tended to have a relative abundance of Lachnospiraceae in excess of $10 \%$, in others the relative abundance was often less than $1 \%$. Variation was found between different human life stages with abundance of Lachnospiraceae highest in the adult $\mathrm{Gl}$ tract, moderate in infants, and approximately $1 \%$ in newborns. Smaller proportions were found in other animals such as fleas and snakes, whose numbers were higher than those of newborn humans and all nonanimal-associated habitats. Only the cow rumen, human digestive tract, human oral cavity, and sewage effluent microbiomes were predicted to have Lachnospiraceae in every sample. As Lachnospiraceae genomes from similar environments were available, extensive functional and phylogenomic analysis of the group was undertaken using 30 representative genomes (supplementary table S2, Supplementary Material online).

\section{Butyric Acid Production Is Not a Defining Trait of the Lachnospiraceae}

Lachnospiraceae members have been implicated in butyric acid production in the human Gl tract (Duncan et al. 2008; Louis et al. 2010; Van-den-Abbeele et al. 2012). It is known that not all members of this family can produce butyrate, raising the question of whether this function was acquired laterally or was ancestrally present and then lost in several lineages. Here, the capability to produce butyric acid along with its evolutionary history was investigated to determine its distribution within the group and the origin of associated genes. Two enzymes allow for the production of butyric acid: butyrate kinase (from Butanoyl-P) (Walter et al. 1993) and 
Table 1

The Distribution of Butyric Acid Production Genes

\begin{tabular}{|c|c|c|}
\hline Name & Butyrate Kinase & BCOAT \\
\hline Anaerostipes caccae DSM 14662 & & + \\
\hline Anaerostipes sp. 3_2_56FAA & & + \\
\hline Butyrivibrio crossotus DSM 2876 & + & \\
\hline Bu. proteoclasticus B316 & + & \\
\hline \multicolumn{3}{|l|}{ Catonella morbi ATCC 51271} \\
\hline \multicolumn{3}{|l|}{ Cellulosilyticum lentocellum DSM 5427} \\
\hline Coprococcus comes ATCC 27758 & + & \\
\hline Co. eutactus ATCC 27759 & + & \\
\hline \multicolumn{3}{|l|}{ Dorea formicigenerans ATCC 27755} \\
\hline \multicolumn{3}{|l|}{ D. longicatena DSM 13814} \\
\hline \multicolumn{3}{|l|}{ Lachnospiraceae bacterium 1_1_57FAA } \\
\hline Lachnospiraceae bacterium 1_4_56FAA & + & \\
\hline \multicolumn{3}{|l|}{ Lachnospiraceae bacterium 2_1_46FAA } \\
\hline \multicolumn{3}{|l|}{ Lachnospiraceae bacterium 2_1_58FAA } \\
\hline \multicolumn{3}{|l|}{ Lachnospiraceae bacterium 3_1_46FAA } \\
\hline Lachnospiraceae bacterium 3_1_57FAA_CT1 & + & \\
\hline \multicolumn{3}{|l|}{ Lachnospiraceae bacterium 4_1_37FAA } \\
\hline \multicolumn{3}{|l|}{ Lachnospiraceae bacterium 5_1_57FAA } \\
\hline Lachnospiraceae bacterium 5_1_63FAA & & + \\
\hline \multicolumn{3}{|l|}{ Lachnospiraceae bacterium 6_1_63FAA } \\
\hline \multicolumn{3}{|l|}{ Lachnospiraceae bacterium 8_1_57FAA } \\
\hline \multicolumn{3}{|l|}{ Lachnospiraceae bacterium 9_1_43BFAA } \\
\hline \multicolumn{3}{|l|}{ Lachnospiraceae oral taxon 107 str. F0167 } \\
\hline \multicolumn{3}{|l|}{ Marvinbryantia formatexigens DSM 14469} \\
\hline \multicolumn{3}{|l|}{ Oribacterium sinus F0268 } \\
\hline \multicolumn{3}{|l|}{ Oribacterium sp. oral taxon 078 str. F0262 } \\
\hline \multicolumn{3}{|l|}{ Oribacterium sp. oral taxon 108 str. F0425 } \\
\hline Roseburia intestinalis L1-82 & & + \\
\hline R. inulinivorans DSM 16841 & & + \\
\hline Shuttleworthia satelles DSM 14600 & + & \\
\hline
\end{tabular}

Note.-The final stage of butyric acid production can be undertaken by two gene groups: butyrate kinase or BCOAT. The presence of each gene within a Lachnospiraceae genome is marked with $a+$.

BCoAT (from Butanoyl-CoA) (Duncan et al. 2002). Only 12 of the 30 sequenced organisms contained genes annotated from at least one of these two pathways (table 1). Pathways appeared to be genus specific as all Shuttleworthia, Butyrivibrio, and Coprococcus genomes encode butyrate kinase and both Roseburia strains, both Anaerostipes strains, and Lachnospiraceae bacterium 5_1_63FAA encode BCoAT. Analysis with TBlastN did not reveal any hits within the other Lachnospiraceae genomes, indicating no related pseudogenes are within these species.

Phylogenetic examination of the two genes revealed evidence of potential LGT. Genes similar to those of the Lachnospiraceae were found within the genomes of several taxa, primarily of the same order as the Lachnospiraceae, the Clostridiales. The topology of each gene tree was tested against a 165 tree derived from the same genomes (supplementary fig. S1, Supplementary Material online). Use of the $\mathrm{AU}$ test (based on the butyrate kinase and BCoAT alignments) showed that the gene trees for butyrate kinase and BCoAT in these species were significantly different from the companion 165 tree $(P<0.001)$. This implies that rearrangements away from a proxy for vertical inheritance occurred within the gene trees, indicative of LGT of both butyrate kinase and BCOAT. Additionally, the $16 \mathrm{~S}$ tree placed many species that are not currently classed in the NCBI taxonomy as Lachnospiraceae (e.g., Eubacterium rectale) proximal to recognized members of this family, suggesting the need for taxonomic revision of the group.

\section{Shared Gene Clusters Reveal Functional Signatures of Habitat Specialization}

A thorough investigation of the family was undertaken to look for defining features of the Lachnospiraceae using sets of homologous gene clusters shared between members of this bacterial family. A total of 167 gene clusters were shared by all sequenced Lachnospiraceae with predicted functions spanning information processing $(46 \%)$, metabolism (15\%, primarily glycolysis and fructose metabolism; COG category G), and cellular processes/signaling ( $9 \%)$, including two multidrug resistance mechanisms and several sigma factors. Thus, only 165 similarity and a handful of metabolic and cellular processes appear to be shared by all members of the Lachnospiraceae family.

Ecological specialization was investigated using pair-wise gene cluster counts between sequenced genomes to observe whether habitat correlated with the presence of specific groups of genes (fig. 2). Some association between habitat and clustering was observed, including a basal split into a group consisting exclusively of 12 human gut-associated family members (referred to as the gut-restricted group) and another group containing genomes from all represented habitats, which contained a smaller cluster of eight gut-associated genomes (fig. 2). The average genome size was 3,539 genes (range: 1,950-6,887) for the mixed habitat group and 2,920 genes (range: $2,081-3,534$ ) for the gut-restricted group. The average genome size within this data set, regardless of clustering, is 3,291, suggesting that group associations are not biased by genome size.

Gene clusters that defined certain groups were investigated further to observe functional patterns. A gene cluster was classed as group specific if it was present in at least $90 \%$ of the genomes in one group and absent from $90 \%$ of the complementary group. Comparison of the gut-restricted group and all other Lachnospiraceae revealed 41 shared gene clusters that were indicative of this group (i.e., present in at least 11 gut-restricted genomes and absent from at least 16 of the other genomes). Functionally, these genes encompassed mostly protein binding (primarily tetratricopeptide repeat motifs), signal transduction, and sporulation, with almost a third of the homologous gene clusters having no annotated function (supplementary table S3a, 


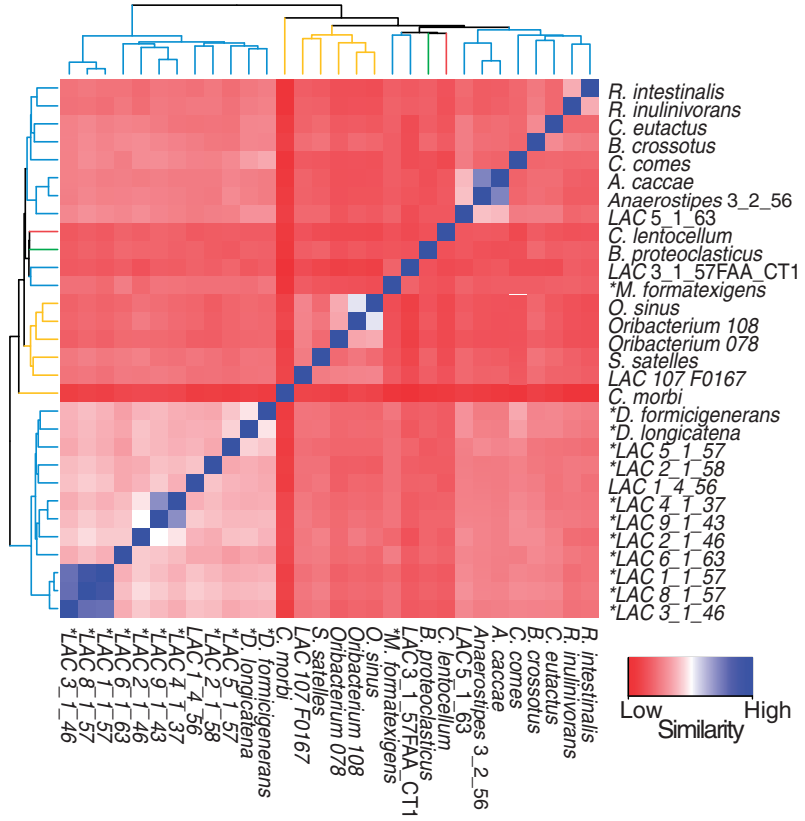

Fig. 2.-Grouping of genomes based on counts of shared gene clusters. Heatmap shows the number of gene clusters shared between genomes, inversely weighted by genome size. Genomes are clustered with intersecting cells between two genomes colored based on similarity ranging from low (red) to high (blue). The hierarchy of clustering is displayed along the side and top of the heat map with branches colored according to habitat (yellow, oral; red, sediment; green, rumen; blue, human GI tract). Names of gut-associated members predicted to be lacking butyric acid production are highlighted by an asterisk.

Supplementary Material online). Only one gene cluster, annotated as an inner membrane component of a transporter complex, was classed as a defining gene cluster for the multihabitat group when compared with the gut-restricted group.

The gut-restricted group was also found to have several gene clusters that distinguish them from the 8 genomes of the other gut-associated Lachnospiraceae (supplementary table S3b, Supplementary Material online). Several tetratricopeptide repeat protein-binding motifs were present in the gut-restricted group and absent from many of the other gut-associated genomes. Most other potentially defining functions encompassed transporters and signaling pathways with $30 \%$ of clusters having no known function. The reverse comparison (clusters absent from the majority of the gutrestricted group but present in the other gut-associated members) revealed several catalytic and transportation-related functions without any discernible pattern.

Almost all of the organisms in the gut-restricted group were also those predicted to be incapable of producing butyric acid (supplementary table S4, Supplementary Material online; fig. 2). This indicates a split in the human gut-associated Lachnospiraceae between those capable of producing butyric acid by either of the known pathways and those who, while lacking this capability, have genomes that are more closely related to each other (the gut-restricted group). Several gene clusters that correlated with the presence or absence of butyric acid production within the human Gl-tract-associated Lachnospiraceae (supplementary table S3C, Supplementary Material online) also distinguished the gut-restricted group from the other gut-associated family members (supplementary tables S3b and table S4, Supplementary Material online). Thus, even though multiple pathways can result in butyric acid production, the presence or absence of this function appears to have an influence on the specialization of certain organisms within the human gut microbiome.

To observe whether similar patterns of distinguishing functions existed between all gut-associated family members (22 strains) and nongut associated members (eight strains), a similar analysis of gene group presence/absence was performed. Fifty-seven functions present in 20 or more gutassociated strains and absent from seven or eight nongutassociated strains were identified (supplementary table S3d, Supplementary Material online). Only one protein was of unknown function with the remaining spread across designations such as DNA binding, repair, and transcription. Several serine-type endopeptidases or associated proteins were present within this group and lacking from the others, suggesting potential involvement of protein modification in adaption to the human Gl tract environment. As was observed with the gut-restricted group, sporulation-related proteins comprised a large fraction of these functions (28\%), although different sporulation proteins distinguished these two groups.

\section{Key Sporulation Proteins Are Detected Only in Human Digestive Tract-Associated Family Members}

We further examined the distribution of four types of sporulation genes: cot genes, which encode protein components of the coat; spo genes, which perform functions across all six stages of sporulation; sps genes, involved in spore coat polysaccharide synthesis; and ssp genes, which create small acid-soluble spore proteins. Homology searches against related sequences from $B$. subtilis (84 genes) revealed that 27 of these genes had no known homolog in any Lachnospiraceae sequenced genome. Of the remaining 57 genes, 29 were present in the majority of gut-associated Lachnospiraceae and completely absent from the rumen and oral-associated family members (fig. 3). These genes were not restricted to any one class or stage of sporulation protein. Cellulosilyticum lentocellum DSM 5427, isolated from sediment containing domestic waste, grouped with the gutassociated members suggesting that it too may be adapted to the human digestive tract.

All sporulation-controlling sigma factors $\left(\sigma^{\mathrm{A}}, \sigma^{\mathrm{E}-\mathrm{H}}\right.$, and $\left.\sigma^{\mathrm{K}}\right)$ were detected in all Lachnospiraceae genomes, which suggests this function was present in the ancestor of the group. 


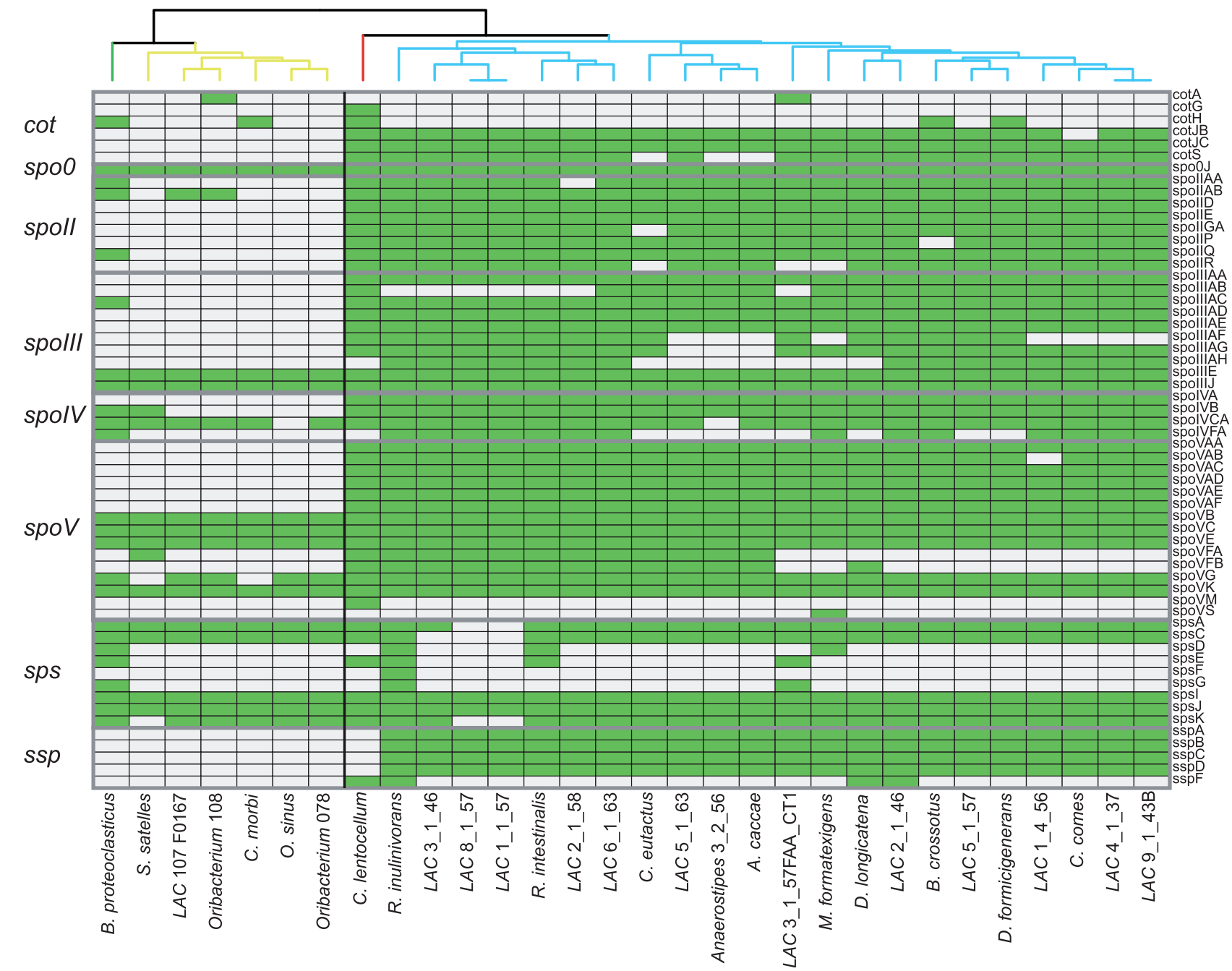

FiG. 3.-Distribution of sporulation-associated genes within Lachnospiraceae genomes. A range of sporulation genes was examined for each genome to assess the capabilities of producing endospores within each strain. Each gene is displayed as present (green) or absent (white) from each Lachnospiraceae genome. Organisms are clustered based on their distribution of sporulation genes. Hierarchical clustering of genomes is displayed at the top of the grid with branches colored according to habitat (yellow, oral; red, sediment; green, rumen; blue, human GI tract). Gray lines separate sporulation genes into the broad categories listed on the right-hand side.

Phylogenetic analysis also suggested vertical transmission of this function, although uncertain taxonomic assignments make such conclusions difficult to confirm. These analyses suggest that gene loss rather than LGT is responsible for the observed habitat-associated pattern of sporulation genes. To confirm a differential presence of sporulation capability in the three habitats (human gut, human oral cavity, and cow rumen), metagenomic samples from each microbiome were mined to find sequences related to each Lachnospiraceaeassociated sporulation protein. Lachnospiraceae-derived sporulation-associated reads were found to be more abundant within the human $\mathrm{Gl}$ tract compared with the cow rumen or human oral cavity $(P<0.001)$ (supplementary fig. S2, Supplementary Material online). The difference in abundance between the rumen and oral cavity was less well supported $(P=0.022$; difference in relative means $=0.013)$. Thus, it is likely that sporulation capabilities within this family are restricted to those found in the human $\mathrm{Gl}$ tract.

\section{Candidate Phylogenies Do Not Reflect Habitat Diversification}

Functional analysis of the Lachnospiraceae-associated genomes revealed both vertical and lateral acquisition of genes that were indicative of subgroups within the family. Although species tree reconstruction can be undertaken in several ways, we chose two popular methods for comparison: 165 phylogeny and a shared ortholog concatenated alignment 
A

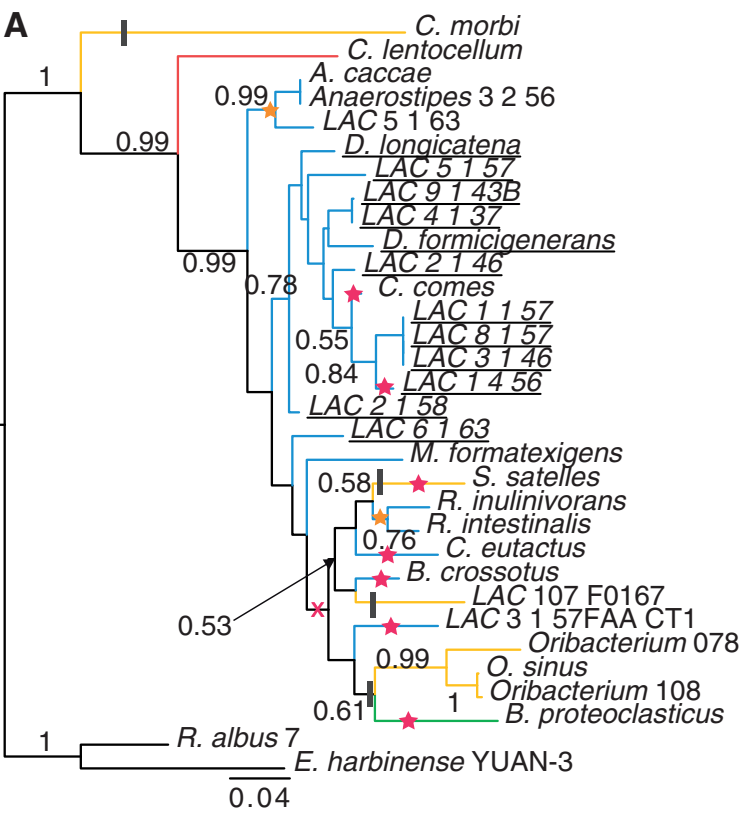

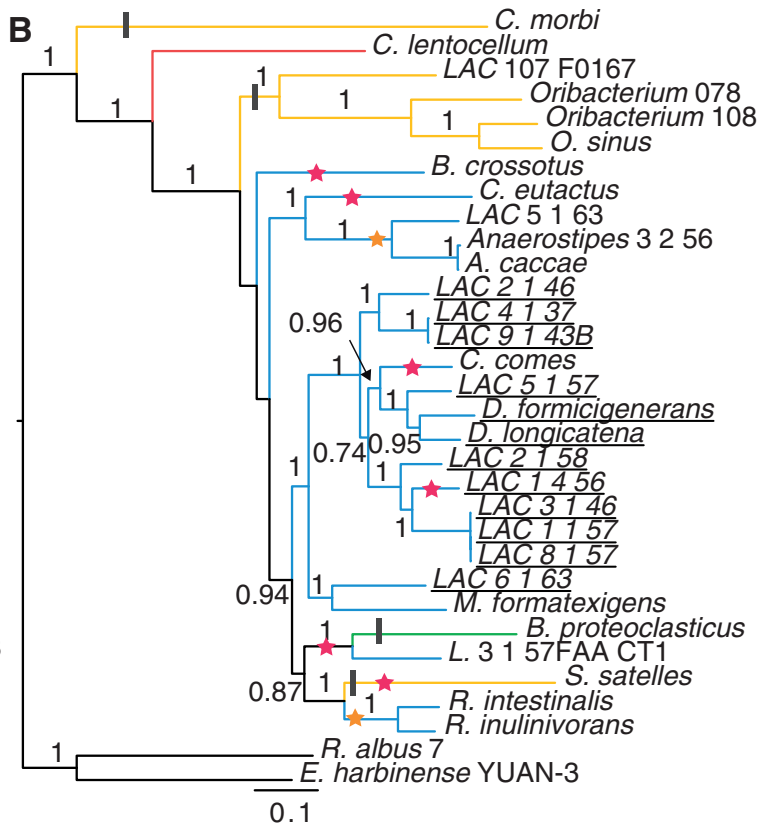

FIG. 4.-Relationships of 30 Lachnospiraceae genomes based on marker gene and concatenated alignments. Phylogenetic trees based on the $16 \mathrm{~S}$ ribosomal RNA gene $(A)$ and the family-wide shared orthologs $(B)$. Trees are rooted using two Ruminococcaceae as outgroup. Branches are colored based on listed habitat (yellow, oral; red, sediment; green, rumen; blue, human GI tract). Bootstrap support values greater than 0.5 are displayed. Locations of putative gain and loss of functions are also shown on the trees. Stars mark the gain of butyric acid production capabilities (pink, butyrate kinase; orange, BCoAT). An alternative gain of butyrate kinase is marked with a pink X on the $16 \mathrm{~S}$ tree (part A). Putative loss of sporulation capabilities is marked with a black bar. Strains classified as gut restricted based on shared gene clusters are underlined.

phylogeny. The copy number of the 16S rRNA gene ranged from 1 (22 genomes) to 11 (one genome) with an average of 2.1 copies per genome. Despite this diversity in copy number, all $16 \mathrm{~S}$ sequences formed clades with respect to their genome. Therefore, only a single representative (the copy with the longest sequence) was retained within the final phylogenies (fig. 4a). The $16 \mathrm{~S}$ tree yielded little support for the majority of clades (38\% of clades with $>75 \%$ bootstrap support) (fig. 4a), likely due to short internal branches in the tree (Wiens et al. 2008). This poor support contrasted with strong support across the tree derived from the concatenated alignment from 91 ubiquitous, single-copy orthologous genes ( $88 \%$ of clades with $>75 \%$ bootstrap support) (fig. $4 b$ ). However, this tree was not in strong agreement with those of the 91 constituent genes according to the AU test (82\% rejected with $P<0.001)$. Even within this restricted "core" set shared by all family members (supplementary table S5, Supplementary Material online), significant phylogenetic discordance is observed. Comparisons of individual core set gene trees revealed low agreement, with only 47 of the 91 gene trees being within an SPR distance of 2 from at least one other shared ortholog tree (supplementary fig. S5, Supplementary Material online). No large core set of genes was found to be in high agreement with each other, suggesting that even core genes are subject to phylogenetic discordance. Comparison of phylogenetic relationships derived from $16 \mathrm{~S}$ sequences and concatenated shared orthologs revealed substantial topological differences, as demonstrated by an SPR distance of 12 between trees with only 30 leaves (supplementary fig. S3, Supplementary Material online). Additionally, each tree was rejected under the AU test $(P<0.001)$ when compared with the alignment of the other (i.e., 16S topology derived from the concatenated alignment and vice versa), demonstrating that neither the $16 \mathrm{~S}$ tree nor the concatenated alignment tree is a convincing proxy for the evolutionary history of the full genomes. The consensus network based on the 91 shared orthologs demonstrated that no clear signal could differentiate the majority of individual strains into a hierarchical structure with little grouping at the genus level, despite high bootstrap support for groupings in the concatenated sequence tree (supplementary fig. S4, Supplementary Material online).

The estimated gain and loss of both butyric acid production and sporulation functionality was mapped onto both the $16 \mathrm{~S}$ and the concatenated sequence trees (fig. 4). Multiple acquisition points of each type of butyric acid production can be observed in both trees, supporting the case for LGT of this function into this group. However, if the $16 \mathrm{~S}$ tree does map the true history of this group, or at least functions as a close proxy for vertical inheritance, the butyrate kinase gene (fig. 4a) 
may have been acquired through LGT by an ancestor of many of the family members and lost in three subsequent lineages, as opposed to five independent gains. This ancient LGT followed by loss in certain lineages is supported by the phylogenetic analysis of this gene, although directionality cannot be determined due to an unresolved species tree (supplementary fig. S1, Supplementary Material online). The observed pattern of sporulation capabilities (fig. 3) could be explained by four gene loss events, no matter the representative tree. This supports a model of vertical inheritance with subsequent gene loss in a habitat-specific manner. Additionally, within the $16 \mathrm{~S}$ tree, most of the gut-restricted group (fig. 2) formed a near clade with one nongroup intruder (Coprococcus comes ATCC 27758) and one member absent (Lachnospiraceae bacterium 6_1_63FAA) (fig. 4).

\section{Discussion}

Lachnospiraceae were found to be present primarily within the mammalian GI tract (fig. 1), as has been suggested previously (Gosalbes et al. 2011; Kittelmann et al. 2013), although low-abundance populations are present in a wider range of environments including nonhost-associated microbiomes. The capacity for butyric acid production, found in fewer than half of the Lachnospiraceae genomes, was not habitat restricted and appears to have been acquired through LGT. Both pathways for producing butyric acid (butyrate kinase and BCOAT) were present in Lachnospiraceae members, with no genome containing both (table 1). Although seven genomes contained butyrate kinase, they appear to have potentially acquired the corresponding gene laterally from other members of class Clostridia (supplementary fig. S1, Supplementary Material online), a group associated with frequent LGT events (Beiko et al. 2005; Sebaihia et al. 2006; Nelson et al. 2010), especially within GI tracts (Meehan and Beiko 2012). LGT has also contributed to the distribution of the BCOAT-mediated pathway, the main route for butyric acid production within the human GI tract (Louis et al. 2004; Louis and Flint 2009). Within both trees, the Lachnospiraceae-related sequences appear to form two clusters, suggesting that the LGT events that gave rise to these functions were likely prior to the speciation events for some of these organisms. Thus, although this function is not habitat-restricted presently, it may have conferred an ecological advantage to the ancestor of some present-day Lachnospiraceae. Determination of the donating partners is difficult in these cases as several species not designated as Lachnospiraceae in the NCBI taxonomy were found in close proximity to organisms such as Roseburia in the 165 phylogeny. An example is E. rectale, which Mannarelli et al. (1990) also placed in family Lachnospiraceae. Such discrepancies between published work and taxonomic databases make determination of directionality and evolutionary history difficult, as the species trees are not well resolved. Reconciling taxonomy and phylogeny is no trivial task given LGT and other challenges but would clarify the origin of butyrate production and other capabilities in the Lachnospiraceae.

Although butyric acid production was not found to segregate the Lachnospiraceae by habitat, several other functions were correlated with specific habitat-associated groups. Tetratricopeptide repeat motif-containing proteins were present in a subset of human Gl tract-associated strains and absent from other members in the same environment (supplementary table S3a and S3b, Supplementary Material online). These motifs play a role in protein-protein interactions and have been associated previously with bacterial pathogens and virulence (Cerveny et al. 2013). As no Lachnospiraceae pathogens have been found before, further investigations into this group, which also lack butyric-acid production capabilities (supplementary table S4, Supplementary Material online), are needed to clarify their role or roles within the human gut.

The ability to produce endospores was found to be a habitat-specific segregating function within the Lachnospiraceae. Genome-wide investigation into the 22 Lachnospiraceae associated with the human $\mathrm{Gl}$ tract revealed an almost full complement of sporulation proteins, whereas those residing in the human oral cavity or cow rumen were lacking such functions (fig. 3, supplementary table S3d, Supplementary Material online). Cellulosilyticum lentocellum, the only Lachnospiraceae with confirmed endospore formation capabilities (Attwood et al. 1996; Kelly et al. 2010), grouped with the Gl tract-associated genomes. This strain was isolated from a sediment bank receiving domestic waste (Murray et al. 1986) and thus may actually be human associated with endospore formation as a habitat adaptation for passage through the human stomach as is observed in C. difficile (Wilson 1983) and cyst formation in several protist species (Bingham and Meyer 1979; Lujan et al. 1997). As analysis of these proteins suggested primarily vertical inheritance of the associated genes, it is likely that this capability was present in a common ancestor and subsequently lost in a habitat-specific fashion.

Our approach to understanding the Lachnospiraceae combined reference genomes of known provenance with marker gene and metagenome samples from a range of habitats. No phylogenomic approach we used produced a separation of lineages based on habitat, raising the question of how lineages can change their habitat preference through time. Discordance between the $16 \mathrm{~S}$ tree and shared ortholog tree indicates that resolution of the "true" species tree may be very difficult for this group. Although a tree based on ribosomerelated genes is often thought to be more accurate for species definitions than 16S alone, the Lachnospiraceae ribosomal protein trees were not in concordance with each other (supplementary fig. S5, Supplementary Material online), suggesting this approach will also give misleading results. We found little support for many genera within this family, and $16 \mathrm{~S}$ trees placed several other organisms within this group (supplementary fig. S1, Supplementary Material online), suggesting 
taxonomic revisions may be required as has been done previously (Moon et al. 2008; Cai and Dong 2010). Despite the inconsistencies observed with regards to taxonomic classifications, some genes clearly separated lineages based on habitat. These genes shed light on how important habitat-specific transitions in the Lachnospiraceae have occurred and how within-habitat divisions, such as the ability to produce butyric acid, can influence the evolution of closely related organisms. As more Lachnospiraceae genomes become available covering important genera such as Blautia and likely mislabeled members such as $E$. rectale, similar analysis may reveal this pattern to extend to these genera and also potentially to other Gl tract-associated microorganisms, revealing how such microbes adapt to the host environment.

\section{Supplementary Material}

Supplementary figures \$1-\$5 and tables \$1-\$5 are available at Genome Biology and Evolution online (http://nww.gbe. oxfordjournals.org/).

\section{Acknowledgments}

The authors thank Dr Petra Louis for supplying the BCOAT database sequences and for discussion of butyrate production. They also thank Morgan Langille, Eva Boon, Katherine Dunn, and W. Ford Doolittle for input and comments. This work was supported by the Canadian Institute for Health Research (grant number CMF-108026) and by Genome Atlantic and the Canada Research Chairs program to R.G.B.

\section{Literature Cited}

Altschul SF, Gish W, Miller W, Myers EW, Lipman DJ. 1990. Basic local alignment search tool. J Mol Biol. 215:403-410.

Attwood GT, Reilly K, Patel BK. 1996. Clostridium proteoclasticum sp. nov., a novel proteolytic bacterium from the bovine rumen. Int J Syst Bacteriol. 46:753-758.

Beiko RG, Harlow TJ, Ragan MA. 2005. Highways of gene sharing in prokaryotes. Proc Natl Acad Sci U S A. 102:14332-14337.

Bingham AK, Meyer EA. 1979. Giardia excystation can be induced in vitro in acidic solutions. Nature 277:301-302.

Brulc JM, et al. 2009. Gene-centric metagenomics of the fiber-adherent bovine rumen microbiome reveals forage specific glycoside hydrolases. Proc Natl Acad Sci U S A. 106:1948-1953.

Bryant MP. 1986. Genus IV. Butyrivibrio. In: Sneath PHA, Mair NS, Sharpe ME, Holt JG, editors. Bergey's manual of systematic bacteriology. Vol. 3. Williams and Wilkins.

Cai S, Dong X. 2010. Cellulosilyticum ruminicola gen. nov., sp. nov., isolated from the rumen of yak, and reclassification of Clostridium lentocellum as Cellulosilyticum lentocellum comb. nov. Int J Syst Evol Microbiol. 60:845-849.

Caporaso JG, et al. 2010. PyNAST: a flexible tool for aligning sequences to a template alignment. Bioinformatics 26:266-267.

Carlier JP, K'Ouas G, Bonne I, Lozniewski A, Mory F. 2004. Oribacterium sinus gen. nov., sp. nov., within the family "Lachnospiraceae" (phylum Firmicutes). Int J Syst Evol Microbiol. 54:1611-1615.

Cerveny L, et al. 2013. Tetratricopeptide repeat motifs in the world of bacterial pathogens: role in virulence mechanisms. Infect Immun. 81:629-635.
Charrier C, et al. 2006. A novel class of CoA-transferase involved in shortchain fatty acid metabolism in butyrate-producing human colonic bacteria. Microbiology 152:179-185.

Cho I, et al. 2012. Antibiotics in early life alter the murine colonic microbiome and adiposity. Nature 488:621-626.

Cole JR, et al. 2009. The Ribosomal Database Project: improved alignments and new tools for rRNA analysis. Nucleic Acids Res. 37:D141-D145.

Criscuolo A, Gribaldo S. 2010. BMGE (Block Mapping and Gathering with Entropy): a new software for selection of phylogenetic informative regions from multiple sequence alignments. BMC Evol Biol. 10:210.

Cryan JF, O'Mahony SM. 2011. The microbiome-gut-brain axis: from bowel to behavior. Neurogastroenterol Motil. 23:187-92.

DeSantis TZ, et al. 2006. Greengenes, a chimera-checked 16S rRNA gene database and workbench compatible with ARB. Appl Env Microbiol. 72:5069-5072.

Downes J, Munson MA, Radford DR, Spratt DA, Wade WG. 2002. Shuttleworthia satelles gen. nov., sp. nov., isolated from the human oral cavity. Int J Syst Evol Microbiol. 52:1469-1475.

Duncan SH, Barcenilla A, Stewart CS, Pryde SE, Flint HJ. 2002. Acetate utilization and butyryl coenzyme A (COA):acetate-CoA transferase in butyrate-producing bacteria from the human large intestine. Appl Environ Microbiol. 68:5186-5190.

Duncan SH, et al. 2008. Human colonic microbiota associated with diet, obesity and weight loss. Int J Obes. 32:1720-1724.

Dworkin M, Falkow S. 2006. The prokaryotes. Bacteria: firmicutes, cyanobacteria a handbook on the biology of bacteria. Vol. 4. Springer.

Edgar RC. 2004a. MUSCLE: a multiple sequence alignment method with reduced time and space complexity. BMC Bioinformatics 5:113.

Edgar RC. 2004b. MUSCLE: multiple sequence alignment with high accuracy and high throughput. Nucleic Acids Res. 32:1792-1797.

Edgar RC. 2010. Search and clustering orders of magnitude faster than BLAST. Bioinformatics 26:2460-2461.

Felsenstein J. 1989. PHYLIP_-Phylogeny Inference Package (Version 3.2). Cladistics 5:164-166.

Gosalbes MJ, et al. 2011. Metatranscriptomic approach to analyze the functional human gut microbiota. PLoS One 6:e17447.

Hague A, Butt AJ, Paraskeva C. 1996. The role of butyrate in human colonic epithelial cells: an energy source or inducer of differentiation and apoptosis? Proc Nutr Soc. 55:937-943.

Hess M, et al. 2011. Metagenomic discovery of biomass-degrading genes and genomes from cow rumen. Science 331:463-467.

Holland BR, Huber KT, Moulton V, Lockhart PJ. 2004. Using consensus networks to visualize contradictory evidence for species phylogeny. Mol Biol Evol. 21:1459-1461.

Huson DH, Bryant D. 2006. Application of phylogenetic networks in evolutionary studies. Mol Biol Evol. 23:254-267.

Kanehisa M, Goto S, Kawashima S, Okuno Y, Hattori M. 2004. The KEGG resource for deciphering the genome. Nucleic Acids Res. 32: D277-D280.

Kelly WJ, et al. 2010. The glycobiome of the rumen bacterium Butyrivibrio proteoclasticus B316(T) highlights adaptation to a polysaccharide-rich environment. PLoS One 5:e11942.

Kinross JM, Darzi AW, Nicholson JK. 2011. Gut microbiome-host interactions in health and disease. Genome Med. 3:14.

Kittelmann S, et al. 2013. Simultaneous amplicon sequencing to explore co-occurrence patterns of bacterial, archaeal and eukaryotic microorganisms in rumen microbial communities. PLoS One 8: e47879.

Kunst $F$, et al. 1997. The complete genome sequence of the gram-positive bacterium Bacillus subtilis. Nature 390:249-256.

Lanfear R, Calcott B, Ho SY, Guindon S. 2012. Partitionfinder: combined selection of partitioning schemes and substitution models for phylogenetic analyses. Mol Biol Evol. 29:1695-1701. 
Lin J, Gerstein M. 2000. Whole-genome trees based on the occurrence of folds and orthologs: implications for comparing genomes on different levels. Genome Res. 10:808-818.

Liu Y, Balkwill DL, Aldrich HC, Drake GR, Boone DR. 1999. Characterization of the anaerobic propionate-degrading syntrophs Smithella propionica gen. nov., sp. nov. and Syntrophobacter wolinii. Int J Syst Bacteriol. 49(Pt 2):545-556.

Louis P, Flint HJ. 2009. Diversity, metabolism and microbial ecology of butyrate-producing bacteria from the human large intestine. FEMS Microbiol Lett. 294:1-8.

Louis P, Young P, Holtrop G, Flint HJ. 2010. Diversity of human colonic butyrate-producing bacteria revealed by analysis of the butyrylCoA:acetate CoA-transferase gene. Env Microbiol. 12:304-314.

Louis $P$, et al. 2004. Restricted distribution of the butyrate kinase pathway among butyrate-producing bacteria from the human colon. J Bacteriol. 186:2099-2106.

Lujan HD, Mowatt MR, Nash TE. 1997. Mechanisms of Giardia lamblia differentiation into cysts. Microbiol Mol Biol Rev. 61:294-304.

Mandal M, Olson DJ, Sharma T, Vadlamudi RK, Kumar R. 2001. Butyric acid induces apoptosis by up-regulating Bax expression via stimulation of the c-Jun N-terminal kinase/activation protein-1 pathway in human colon cancer cells. Gastroenterology 120:71-78.

Mannarelli BM, Stack RJ, Lee D, Ericsson L. 1990. Taxonomic relatedness of Butyrivibrio, Lachnospira, Roseburia, and Eubacterium species as determined by DNA hybridization and extracellular-polysaccharide analysis. Int J Syst Bacteriol. 40:370-378.

Matsen FA, Kodner RB, Armbrust EV. 2010. pplacer: linear time maximum-likelihood and Bayesian phylogenetic placement of sequences onto a fixed reference tree. BMC Bioinformatics 11:538.

Mclntyre A, Gibson PR, Young GP. 1993. Butyrate production from dietary fibre and protection against large bowel cancer in a rat model. Gut 34 386-391.

McLellan SL, et al. 2013. Sewage reflects the distribution of human faecal Lachnospiraceae. Env Microbiol. 15(8):2213-2227.

Meehan CJ, Beiko RG. 2012. Lateral gene transfer of an ABC transporter complex between major constituents of the human gut microbiome. BMC Microbiol. 12:248.

Meyer F, et al. 2008. The metagenomics RAST server-a public resource for the automatic phylogenetic and functional analysis of metagenomes. BMC Bioinformatics 9:386.

Moon CD, et al. 2008. Reclassification of Clostridium proteoclasticum as Butyrivibrio proteoclasticus comb. nov., a butyrate-producing ruminal bacterium. Int J Syst Evol Microbiol. 58:2041-2045.

Muegge BD, et al. 2011. Diet drives convergence in gut microbiome functions across mammalian phylogeny and within humans. Science 332 970-4.

Murray WD, Hofmann L, Campbell NL, Madden RH. 1986. Clostridium lentocellum sp. nov., a cellulolytic species from river sediment containing paper-mill waste. Syst Appl Microbiol. 8:181-184.

Nelson DM, Cann IK, Altermann E, Mackie RI. 2010. Phylogenetic evidence for lateral gene transfer in the intestine of marine iguanas. PLoS One 5 e10785.

Nepelska M, et al. 2012. Butyrate produced by commensal bacteria potentiates phorbol esters induced AP-1 response in human intestinal epithelial cells. PLoS One 7:e52869.

Newton RJ, Vandewalle JL, Borchardt MA, Gorelick MH, McLellan SL. 2011. Lachnospiraceae and Bacteroidales alternative fecal indicators reveal chronic human sewage contamination in an urban harbor. Appl Env Microbiol. 77:6972-6981.

Parks DH, Beiko RG. 2010. Identifying biologically relevant differences between metagenomic communities. Bioinformatics 26:715-21.
Petrof EO, et al. 2013. Stool substitute transplant therapy for the eradication of Clostridium difficile infection: "RePOOPulating" the gut. Microbiome 1:3.

Price MN, Dehal PS, Arkin AP. 2010. FastTree 2-approximately maximum-likelihood trees for large alignments. PLoS One 5:e9490.

Pryde SE, Duncan SH, Hold GL, Stewart CS, Flint HJ. 2002. The microbiology of butyrate formation in the human colon. FEMS Microbiol Lett. 217:133-139.

Roediger WE. 1980. Role of anaerobic bacteria in the metabolic welfare of the colonic mucosa in man. Gut 21:793-798.

Sayers EW, et al. 2010. Database resources of the National Center for Biotechnology Information. Nucleic Acids Res. 38:D5-D16.

Sebaihia $M$, et al. 2006. The multidrug-resistant human pathogen Clostridium difficile has a highly mobile, mosaic genome. Nat Genet. 38:779-786

Shimodaira H, Hasegawa M. 2001. CONSEL: for assessing the confidence of phylogenetic tree selection. Bioinformatics 17:1246-1247.

Smillie CS, et al. 2011. Ecology drives a global network of gene exchange connecting the human microbiome. Nature 480:241-244.

Stamatakis A. 2006. RAxML-VI-HPC: maximum likelihood-based phylogenetic analyses with thousands of taxa and mixed models. Bioinformatics 22:2688-2690.

Sun CQ, et al. 1998. The effect of $\mathrm{pH}$ on the inhibition of bacterial growth by physiological concentrations of butyric acid: implications for neonates fed on suckled milk. Chem Biol Interact. 113:117-131.

Tatusov RL, Galperin MY, Natale DA, Koonin EV. 2000. The COG database: a tool for genome-scale analysis of protein functions and evolution. Nucleic Acids Res. 28:33-36.

Turnbaugh PJ, et al. 2008. A core gut microbiome in obese and lean twins. Nature 457:480-484

Van-den-Abbeele P, et al. 2012. Butyrate-producing Clostridium cluster XIVa species specifically colonize mucins in an in vitro gut model. ISME J. 7:949-961.

Walter KA, Nair RV, Cary JW, Bennett GN, Papoutsakis ET. 1993. Sequence and arrangement of two genes of the butyrate-synthesis pathway of Clostridium acetobutylicum ATCC 824. Gene 134: 107-111.

Warnes GR, Bolker B, Lumley T. 2012. gplots: Various R programming tools for plotting data. R package version 2.6.0. [cited 2014 Mar 14]. Available from: http://cran.r-project.org/web/packages/gplots/.

Whidden C, Beiko RG, Zeh N. 2010. Fast FPT algorithms for computing rooted agreement forests: theory and experiments. In: Experimental Algorithms, 9th International Symposium. SEA 2010. Ischia Island, Naples (Italy): Springer-Verlag. p. 141-153.

Wiens JJ, et al. 2008. Branch lengths, support, and congruence: testing the phylogenomic approach with 20 nuclear loci in snakes. Syst Biol. 57: 420-431.

Wilson KH. 1983. Efficiency of various bile salt preparations for stimulation of Clostridium difficile spore germination. J Clin Microbiol. 18: 1017-1019.

Yatsunenko T, et al. 2012. Human gut microbiome viewed across age and geography. Nature 486:222-227.

Zdobnov EM, Apweiler R. 2001. InterProScan—an integration platform for the signature-recognition methods in InterPro. Bioinformatics 17: 847-848.

Zeng AP, et al. 1994. Multiple product inhibition and growth modeling of Clostridium butyricum and Klebsiella pneumoniae in glycerol fermentation. Biotechnol Bioeng. 44:902-911.

Associate editor: Tal Dagan 